\title{
High-Sensitivity Boron Nitride Sputter Erosion Measurements by Continuous-Wave Cavity Ring-Down Spectroscopy
}

\author{
Azer P. Yalin", Lei Tao ${ }^{\dagger}$, Ryan Sullenberger \\ Colorado State University, Fort Collins, Colorado, 80523 USA \\ Masashi Oya ${ }^{\S}$, Naoji Yamamoto** \\ Kyushu University, Kasuga, Japan \\ Timothy B. Smith ${ }^{\dagger \dagger}$, Alec D. Gallimore ${ }^{\sharp \dagger}$ \\ University of Michigan, Ann Arbor, Michigan, 48109 USA
}

\begin{abstract}
Sputter erosion of boron nitride $(\mathrm{BN})$ plays a key life-limiting role in many Hall thrusters. We report on the development of cavity ring-down spectroscopy (CRDS) as a sensitive and accelerated diagnostic for sputter erosion. The measurement approach is based upon probing sputtered boron atoms in the region of $250 \mathrm{~nm}$. We present quantitative detection of sputtered BN using CRDS with a pulsed laser source. The pulsed measurement results are compared with modeled signal levels. We update the development of a continuous-wave CRDS system using the frequency-quadrupled output of an external cavity diode laser as the light source. Characterizations of the measurement sensitivity show that with integration times of several minutes, the current performance would allow high signal-to-noise erosion measurements for expected thruster conditions. Approaches to increase sensitivity (reduce measurement time) and to integrate with vacuum chambers for thruster testing are summarized.
\end{abstract}

\section{Nomenclature}

$\begin{array}{lll}A_{k i} & = & \text { Einstein } A \text { coefficient, } 1 / \mathrm{s} \\ A b s & = & \text { Absorbance } \\ A b s_{\text {Min }}= & \text { Minimum Detectable Absorbance } \\ c & =\text { Speed of light, } 2.998 \times 10^{8} \mathrm{~m} / \mathrm{s} \\ E_{B} & =\text { Binding energy }(\mathrm{eV}) \\ g & =\text { Cavity g-parameter }(=1-l / R O C) \\ g_{i} & =\text { Degeneracy of state } i \\ g_{k} & =\text { Degeneracy of state } k \\ k(v) & =\text { Absorption coefficient, } \mathrm{m}^{-1} \\ l & =\text { Length of the ring-down cavity, } \mathrm{m} \\ n & =\text { Exponent in Thomson velocity distribution } \\ N_{i} & =\text { Lower state concentration, } \mathrm{m}^{-3} \\ R & =\text { Mirror reflectivity } \\ R O C & =\text { Mirror radius-of-curvature, } \mathrm{m} \\ S(t, v) & =\text { Ring-down signal }\end{array}$

\footnotetext{
*Associate Professor, Mechanical Engineering, ayalin@engr.colostate.edu

${ }^{\dagger}$ Graduate Student, Mechanical Engineering

${ }^{*}$ Undergraduate Student, Mechanical Engineering

${ }^{\S}$ Graduate Student, Department of Advanced Energy Engineering Science

** Assistant Professor, Department of Advanced Energy Engineering Science

${ }^{\dagger \dagger}$ Post-Doctoral Researcher, Aerospace Engineering

Professor, Aerospace Engineering
} 


$\begin{array}{ll}Y & =\text { Total sputter yield, atoms/ion or } \mathrm{mm}^{3} / \mathrm{C} \\ v & =\text { Laser frequency, } \mathrm{Hz} \\ v_{k i} & =\text { Transition frequency, } 1 / \mathrm{s} \\ \tau & =\text { Ring-down time, } \mathrm{s} \\ \tau_{0} & =\text { Empty cavity ring-down time, } \mathrm{s}\end{array}$

\section{Introduction}

he lifetimes of Hall thruster devices, especially stationary plasma thrusters (SPTs), tend to be limited by sputter erosion of boron nitride (BN) from the acceleration channel. Ideally this problem would be addressed with both numerical modeling and direct lifetime measurements. Research in the former area is underway and generally involves expanding thruster codes to include sputter erosion by considering ion fluxes to the wall and the resulting sputtering $^{1-3}$. Of course, prediction of the sputtering depends critically on the sputter yield parameters used in the code. A key challenge is the paucity of basic sputter yield data at the needed conditions, i.e. for sputtering of boron nitride by xenon ions, especially for the low ion energies $(\sim 100 \mathrm{eV}$ and below) thought to cause much of the erosion. Related research being conducted in our laboratory is focused on exactly such measurements ${ }^{4}$. On the experimental side, direct lifetime measurements are also very challenging. With proposed thrust durations now as long as 5-10+ years, ground-based life tests over the full thruster duration are becoming increasingly expensive and limiting in terms of technology insertion schedules. For example, an SPT-100 was operated for over 5,700 hours and performed nearly 7,000 start cycles at JPL as part of the engine's flight qualification process ${ }^{5}$. JPL also tested two ion thrusters continuously for 8,000 and 12,000 hours ${ }^{6}$. Clearly such approaches have numerous limitations including high cost and time intensiveness as well as only the possibility of post-facto bulk analysis as opposed to real time analysis as operating points are varied.

The ideal diagnostic for in situ thruster studies should have high sensitivity to measure low erosion rates, the possibility of integration to a thruster test-facility, and fast time-response to explore a range of operating conditions. Techniques, such as weight loss $s^{4,7}$, collector plates ${ }^{8-9}$, quartz crystal microbalance ${ }^{4,10-12}$, radioactive tracers ${ }^{13}$, mass spectrometry $^{14}$, and Rutherford backscattering ${ }^{15-16}$ each have certain advantages and can be appropriate for material sputter characterization studies, but none readily meets all of the above criteria. The need for a sensitive nonintrusive measurement suggests the use of optical techniques. Optical emission spectroscopy $(\mathrm{OES})^{17-18}$, laser induced fluorescence (LIF) ${ }^{19-21}$, and multi-photon ionization coupled to a time of flight mass spectrometer ${ }^{22-24}$ have all been used for species-specific sputter yield measurements. The use of LIF has been particularly extensive, and has proven to be very effective for velocity measurement though challenging for quantitative number density. OES spectroscopy is attractive owing to its experimental simplicity but the analysis can be challenging since collisionalradiative modeling is required to extract number densities. OES has also been used for in situ thruster erosion measurements but again with rate assumptions or rate modeling required ${ }^{25-27}$.

In contrast to these techniques, our approach is to develop a sputter diagnostic based on cavity ring-down spectroscopy (CRDS). CRDS is a highly sensitive laser-based absorption method allowing species-specific measurement of concentrations of trace species. Past work at Colorado State University (CSU) has shown the use of pulsed-CRDS to quantitatively measure sputter products ${ }^{28-32}$. We view that CRDS can function both as a method for sputter yield measurement and material characterization, to generate inputs for erosion and lifetime modeling; and can also provide an in situ diagnostic suitable to study thruster erosion and lifetime and aid in thruster design ${ }^{32}$. Development on an in situ sputter sensor is underway in a collaborative effort between researchers at CSU, University of Michigan, and Kyushu University.

This contribution is laid out as follows. Section II of this paper we review the theory of CRDS and introduce the scheme for measuring BN. The approach is to detect sputtered boron atoms in the vicinity of $250 \mathrm{~nm}$ in the ultraviolet. In Section III we present results of pulsed-CRDS experiments measuring BN targets. In Section IV we update the development of CRDS based on a continuous-wave (cw) lasers for enhanced sensitivity. Conclusions and a summary are presented in Section V. 


\section{Cavity Ring-Down Spectroscopy (CRDS) Detection of Sputtered Boron Nitride}

\section{A. Cavity Ring-Down Spectroscopy (CRDS)}

CRDS is a highly sensitive laser-based absorption technique ${ }^{33,34}$ that is directly quantifiable and well suited to measurements of low concentrations of sputtered particles. It is an absorption technique so that ground states are directly measurable. Measuring ground states can be advantageous since these energy levels typically contain a large fraction of the overall species population and their population fractions are minimally affected by collisional and radiative rates. (This is contract to OES approaches that probe the small fraction of the sputtered particles that are in their upper energy-levels, and for which the relation of the upper-level population fraction to overall number density requires rate modeling.) The main practical impediment in applying CRDS is the need to study optically accessible species, i.e. to work at wavelengths where optical sources, detectors, and high-reflectivity mirrors are available. In terms of signal analysis, the path-integrated nature of CRDS can be challenging and may require the use of inversion (tomographic) techniques to recover spatially resolved profiles ${ }^{31}$.

As shown in Fig. 1, the basic idea is to house the absorbing sample (i.e. the sputtered atoms) within a highfinesse optical cavity formed from high-reflectivity mirrors. The interrogating laser beam is coupled into the optical cavity where it "bounces" many times back-and-forth between the mirrors. Owing to the high reflectivity, the light within the cavity makes many passes within the cavity. A detector placed behind the cavity measures the temporal decay of optical intensity within the cavity. The difference in the temporal decay rate with and without the absorber (or with the laser tuned on/off the resonance) yields the sample concentration. Sensitivity of CRDS is largely due to the enhanced cavity length and which is inversely proportional to mirror loss. In the visible, near infrared, and near ultraviolet, mirrors with reflectivity better than 0.9999 (loss less than $100 \mathrm{ppm}$ ) are readily available. As will be further discussed, a challenge for the BN detection is that measurements are made at $\sim 249 \mathrm{~nm}$, and in this region available mirrors are limited to reflectivity $<\sim 0.997$.

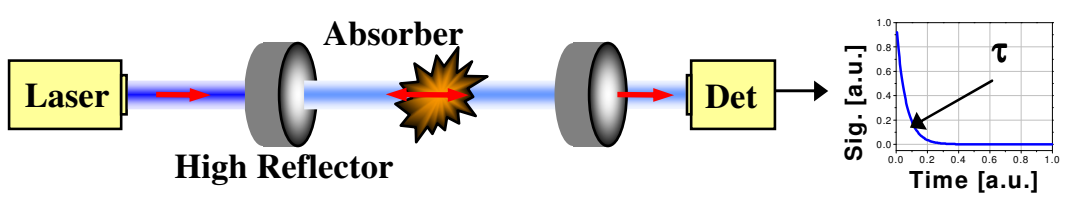

Figure 1. Schematic diagram of cavity ring-down spectroscopy (CRDS).

Under appropriate conditions, the ring-down signal $S(t, v)$ decays single exponentially ${ }^{35,36}$ versus time as:

$$
\begin{aligned}
& S(t, v)=S_{0} \exp [-t / \tau(v)] \\
& 1 / \tau(v)=\frac{\mathrm{c}}{l}\left[\int k(x, v) d x+(1-R)\right]
\end{aligned}
$$

where $\tau$ is the $1 / \mathrm{e}$ time of the decay (termed the ring-down time), $c$ is the speed of light, $l$ is the cavity length, $k(x, v)$ is the absorption coefficient, $v$ is the laser frequency, $x$ is the position along the optical axis, and 1- $R$ is the effective mirror loss (including scattering and all cavity losses). If the absorber is uniformly present over a column length $l_{a b s}$, then $\int k(x, v) d x$ can be replaced with the product $k(v) l_{a b s}$. In practice, the measured ring-down signal is fitted with an exponential, and the ring-down time $\tau$ is extracted. Combining $\tau$ with the "empty cavity ring-down time", $\tau_{0}$ (which in practice is measured by detuning the laser) allows determination of the sample absorbance, Abs, and absorption coefficient:

$$
A b s(v) \equiv \int k(x, v) d x=\frac{l}{c}\left[\frac{1}{\tau(v)}-\frac{1}{\tau_{0}}\right]
$$


A commonly used approach is to scan the laser frequency across the absorption line and to measure the wavelength- (or frequency-) integrated spectrum, i.e. the line area. Assuming the spectroscopic line parameters are known, the measured area $\int A b s(v) d v$ of a transition from lower state $i$ to upper state $k$ can be readily converted to the path-integrated concentration of the lower state $\int N_{i} d x$ as:

$$
\int N_{i} d x=8 \pi \frac{g_{i}}{g_{k}} \frac{v_{k i}^{2}}{A_{k i} c^{2}}\left(\int A b s(v) d v\right)
$$

where $g_{i}, g_{k}$ are the level degeneracies, $v_{k i}$ is the transition frequency, $A_{k i}$ is the transition Einstein $A$ coefficient, and $c$ is the speed of light. For cases where the spatial distribution of particles is non-uniform, actual concentration profiles can be determined from the path-integrated concentration in several ways. For rough approximation one can assume a uniform concentration profile over a known column length, $l_{a b s}$. Alternatively, Abel inversion or shooting techniques with modeled spatial profiles can be used ${ }^{31}$. CRDS can also be used to extract velocity information from the measured spectral lineshapes ${ }^{30}$, which for sputtered particles corresponds to information on the material binding energy, $E_{b}$, since $E_{b}$ governs the Thomson velocity distribution.

The presentation in this section has assumed a decaying ring-down signal as would be the case when using pulsed lasers. As will be discussed in Section IV, we are currently developing a CRDS sensor based on a continuous-wave (cw) laser. For the cw-CRDS implementation the concept is similar; however, the input beam must be extinguished with a modulator in order to produce the decaying ring-down signal.

\section{B. CRDS Detection of Sputtered Boron Nitride}

The detection approach for BN has been presented in our past work ${ }^{32}$. Briefly, the CRDS absorption measurement is based on probing sputtered boron atoms. Table 1 shows detailed energy levels, spontaneous emission rates (Einstein A coefficients), and wavelengths for the targeted lines. Line parameters, especially $A_{k i}$ coefficients differ by about $20 \%$ between the NIST and Kurucz Atomic Line Database. Table 1 uses values from the latter, while we average the two in our current analyses. The ground term for this species has two distinct levels: $2 s^{2} P_{1 / 2}^{0}$ (at $0.00000 \mathrm{eV}$ ) and $2 s^{2} P_{3 / 2}^{0}$ (at $0.00189 \mathrm{eV}$ ). As a result, fine-structure splitting results in two distinct B I absorption lines near $250 \mathrm{~nm}$ : the $2 s^{2} P_{1 / 2}^{0} \rightarrow 3 s{ }^{2} S_{1 / 2}$ absorption at $249.753 \mathrm{~nm}$, and the $2 s{ }^{2} P_{3 / 2}^{0} \rightarrow 3 s{ }^{2} S_{1 / 2}$ absorption at $249.848 \mathrm{~nm}$. The targeted lines are selected based on their optical accessibility and high absorption strength. The next lowest energy level is above $3 \mathrm{eV}$ so that to a good approximation all population will reside in the split ground state, meaning that the measured states will provide a direct measure of the overall boron population. For a rough estimate of expected signal strengths we have simulated absorbance spectra. For example, a boron number density of $10^{8} \mathrm{~cm}^{-3}$ over a path length of $10 \mathrm{~cm}$ (path integrated concentration of $10^{9} \mathrm{~cm}^{-2}$ ) yields a peak optical absorbance of $\sim 30 \mathrm{ppm}$ assuming a linewidth similar to that in our past measurements $(\sim 0.007 \mathrm{~nm})$.

\begin{tabular}{|c|c|c|c|}
\hline$E_{i}\left(\mathrm{~cm}^{-1}\right)$ & $E_{k}\left(\mathrm{~cm}^{-1}\right)$ & $A_{k i}\left(\mathrm{~s}^{-1}\right)$ & $\lambda(\mathrm{nm})$ \\
\hline 0 & 40039.52 & $9.552 \times 10^{7}$ & 249.753 \\
\hline 15.15 & 40039.52 & $1.904 \times 10^{8}$ & 249.848 \\
\hline
\end{tabular}

Table 1. Spectroscopic information for boron (from Kurucz Atomic Line Database).

\section{BN Measurements by Pulsed-CRDS}

Our past work has included proof of principle experiments to detect boron using pulsed CRDS $^{32}$. Measurements were conducted in a diagnostic chamber at sputtering conditions significantly higher than expected for a Hall thruster. Here, we build upon that work as a means to better characterize the detection system. The quantitative measurements also allow comparison with a sputter model and measurements of BN sputter yields. 


\section{A. Experimental}

Figure 2 shows a schematic diagram of the bench-top sputtering apparatus employed for CRDS diagnostic development ${ }^{28-32}$. The apparatus allows the use of CRDS to probe sputtered particles created by an ion beam incident upon a target. The key components are an ion beam and target, housed within a vacuum facility. A roughing and turbo-pump (Turbo-V550) are used to bring the pressure to approximately $10^{-6}$ torr under no-flow conditions. A small argon flow $(2.05 \mathrm{sccm})$ is used to feed the system. The ion beam is extracted from a structurally integrated thruster obtained from NASA, and modified to operate on an inert gas, and to use refractory metal filaments for both the main and neutralizer cathodes in place of the hollow cathodes used in the original design. The 8-cm diameter ion source has been masked, so that the beam leaving the source has a dimension of 8 $\mathrm{cm} \times 2.5 \mathrm{~cm}$, with the $8-\mathrm{cm}$ dimension parallel to the optical axis. The thruster operates with an IonTech power supply (MPS 3000).

The pulsed CRDS set-up uses a broadly tunable optical parametric oscillator (OPO) laser. For the boron experiments we use the doubled-signal beam as the light source in the $250 \mathrm{~nm}$ region. Laser parameters are: repetition rate $=10 \mathrm{~Hz}$, pulse width $\sim 7 \mathrm{~ns}$, pulse energy $\sim 2 \mathrm{~mJ}$, and linewidth $\sim 0.002 \mathrm{~nm}$. We use a linear ringdown cavity of $84 \mathrm{~cm}$ length with $100 \mathrm{~cm}$ radius-of-curvature (ROC) mirrors $(g=0.16)$. The ring-down signal is collected behind the output mirror with a fast photomultiplier tube (Hamamatsu R9110). The spectra reported below use a wavelength step-size of $0.001 \mathrm{~nm}$ and generally average 72 ring-down signals (7.2 s) at each wavelength step. Ring-down signals are fit between $90 \%-10 \%$ of the peak amplitude. We use area (frequency-integrated) measurements of absorbance in our analyses. As mentioned in Section II, a challenge in our spectral region is the limited reflectivity of available mirrors and we use mirrors with $\mathrm{R} \approx 0.992-0.995$. A future approach based on prismbased reflectors is discussed in Section IV.

The pulsed-CRDS tests have measured a $15 \mathrm{~cm}$ x $14 \mathrm{~cm}$ sample of HBR grade BN from General Electric's (GE's) Advanced Ceramics. The material is formed by hot-pressing and corresponds to the graphite-like allotrope of BN. In the base plane, atoms are held together by strongly directed hexagonal arrays of covalent bonds, resulting in impressive electrical, thermal, and mechanical properties. The HBC BN uses a calcium borate binder.

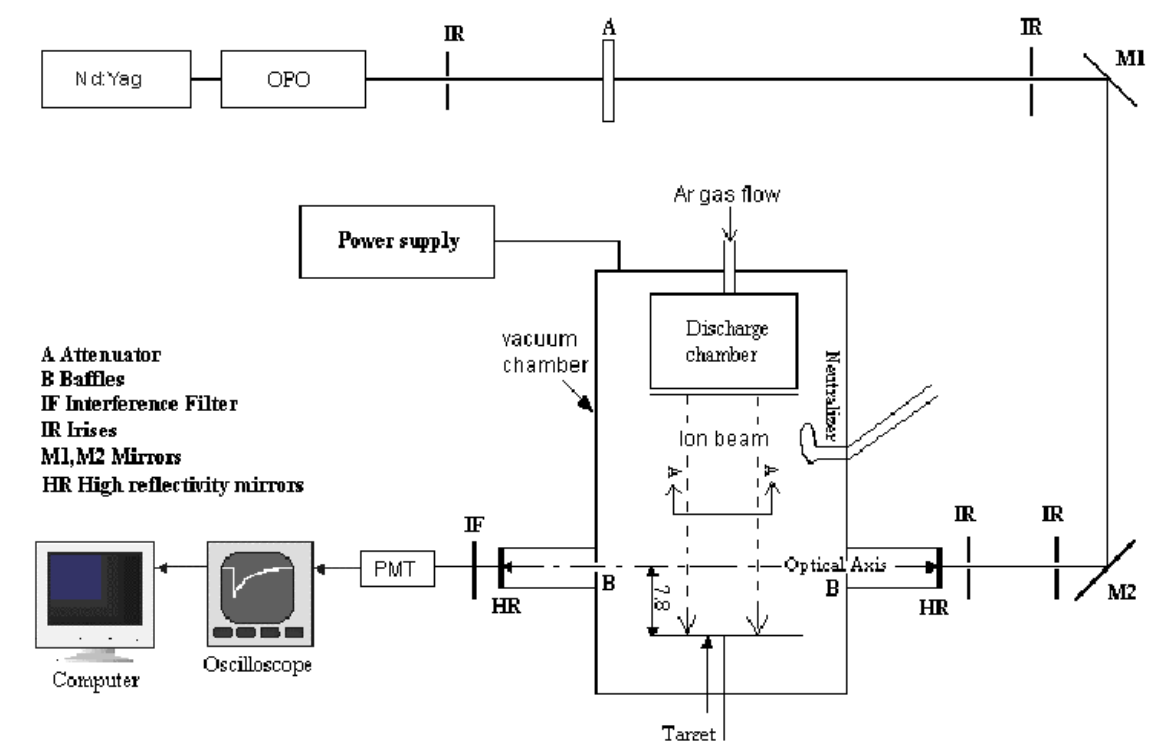

Figure 2. Schematic diagram of sputtering apparatus and CRDS system.

\section{B. Results and Discussion}

Prior to measuring BN, we have validated our CRDS sputter measurement capability by studying the sputtering of aluminum by argon ions. We have used a rectangular aluminum target $15 \mathrm{~cm} \mathrm{x} 11 \mathrm{~cm}$ with the laser beam centered over the target at a height of $1.5 \mathrm{~cm}$ above the target.. The ion source was operated on argon and 
measurements were made for an ion energy of $1000 \mathrm{eV}$ and beam current of $33 \mathrm{~mA}$. The number density was found by integrating the areas of the absorption feature at $396.152 \mathrm{~nm}$. We have found good agreement between the measured path-integrated concentration, $3.17 \pm 0.10 \times 10^{10} \mathrm{~cm}^{-2}$, and the associated modeled value $4.0 \pm 1.2 \times 10^{10}$ $\mathrm{cm}^{-2}$. The modeling approach uses the finite-element scheme described in previous work ${ }^{29-31}$ and discussed later in this section. In this case, the model used the measured current distribution of the source and assumes: $Y=1.2$ atoms/ion, $E_{b}=3.36 \mathrm{eV}$ (for $\mathrm{Al}$ ), $n=1.8$ (exponent on velocity distribution, see References 29-31 and therein), a diffuse differential sputter yield, and spectroscopic constants for Al from NIST database. The uncertainty on the experimental value is from reproducibility of the measurements, while the uncertainty in the modeled value $( \pm 31 \%)$ has contributions from the total yield $( \pm 10 \%)$, shape of differential sputter yield $( \pm 15 \%)$, velocity distribution $( \pm$ $20 \%$ ), and current density distribution $( \pm 15 \%)$. The agreement between measurement and model is taken as a basic validation of our measurement system.

The left of Figure 3 shows representative CRDS data from the HBR grade BN sample for the $249.848 \mathrm{~nm}$ line. Measurements use a $15 \mathrm{~cm} \times 14 \mathrm{~cm}$ target of $\mathrm{HBC}$ grade of BN with ion energy of $800 \mathrm{eV}$, ion beam current of 30 $\mathrm{mA}$, and with the optical axis $1.0 \mathrm{~cm}$ above the target. The displayed spectrum is as directly measured, with ringdown time versus wavelength. The right of Figure 3 shows the same spectrum converted to (dimensionless) optical absorbance and after baseline subtraction. As described in our previous work ${ }^{29-31}$, the shape, or width, of the measured lineshape is due to Doppler shifting from the particle velocities. By using a "shooting method" we compare the measured lineshape with modeled values corresponding to different Thomson velocity distribution. The model uses a finite element approach. The target is divided into a series of elements as is the optical axis. Frequency-resolved contributions, based on Doppler shifting associated with the particle's Thomson velocity distribution, are determined between each point on the target and optical axis. The amplitude of each contribution is found from the concentration of sputtered particles at the corresponding location along the optical-axis due to the corresponding location on the target, which is found from the geometry, measured current density distribution on the target, differential sputter yield distribution (which we assume to be diffuse), and Thomson velocity distribution. The lineshape contributions are added together to determine the expected overall lineshape. The Thomson distributions are specified by an exponent, $n$, for which we use $n=1.8$ as in much of our past work, the mass of the ejected particle, and the binding energy, $E_{b}$. For an initial estimate of the binding energy of $\mathrm{BN}$ we take $E_{b}=3.865$ $\mathrm{eV}$ based on averaging the values for $\mathrm{B}$ and $\mathrm{N}$. Included in the right of Figure 3 is a modeled lineshape curve based on this value of $\left.E_{b}\left(V_{b}=\left(2 E_{b} / M\right)^{0.5}=8300 \mathrm{~m} / \mathrm{s}\right)\right)$. Noise in the data limits our ability to accurately fit for Eb. We will repeat such measurements with the higher sensitivity cw-CRDS system. For now, we find that $E_{b}=3.865 \mathrm{eV}$ agrees reasonably well with the measured lineshape and thus provides an initial rough estimate.
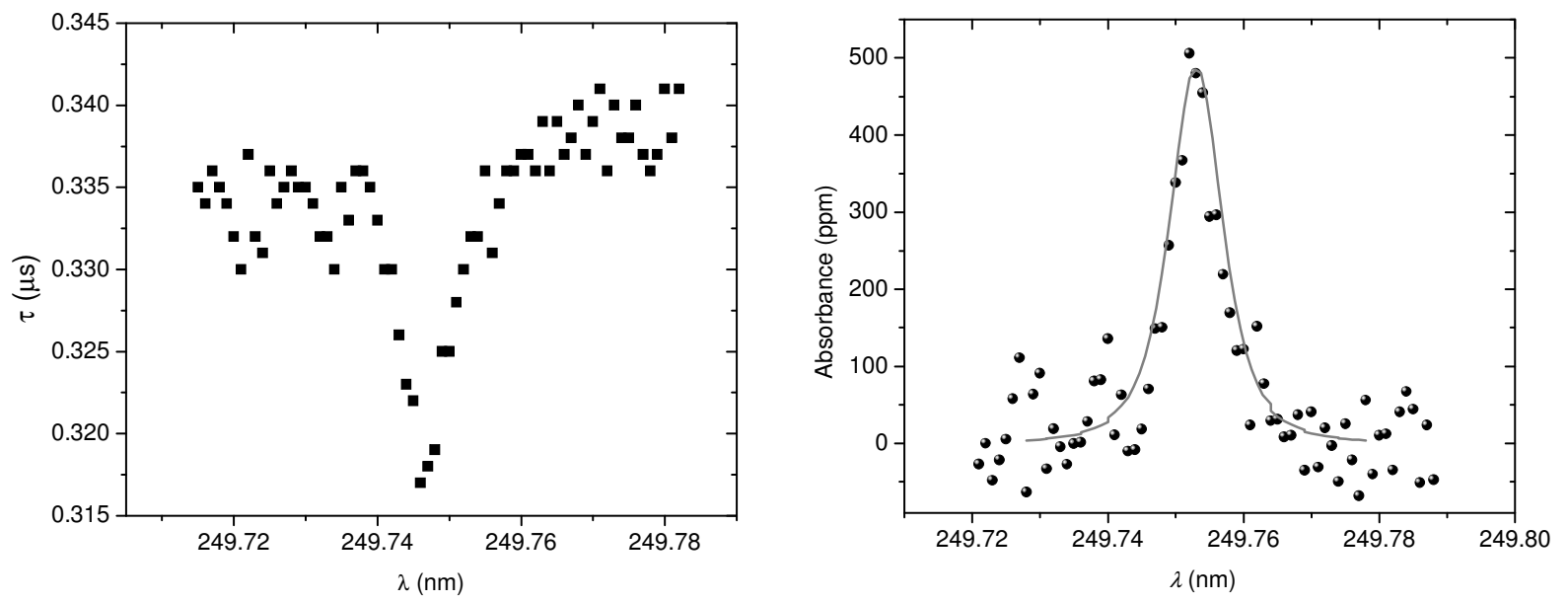

Figure 3. CRDS measurement of boron from BN target. Left: Raw data. Right: CRDS absorbance spectrum and modeled lineshape based on $E_{b}=3.865 \mathrm{eV}$ (see text).

We have performed a study of dependence of boron number density versus beam voltage. Using similar modeling to that described above, we can infer the boron sputter yield from the measured number density. We again use the measured current density distribution on the target, assume a diffuse shaped sputter yield, and adopt $E_{b}=$ $3.865 \mathrm{eV}$ and $n=1.8$ in Thomson distribution. The uncertainty in the CRDS-based sputter yield is due to uncertainties in: Einstein A coefficient $( \pm 11 \%)$, shape of differential sputter yield $( \pm 5 \%)$; velocity distribution $( \pm$ 
$21 \%)$, current density distribution ( $\pm 10 \%$ ), and reproducibility of CRDS measurements. We first find the sputter yield in atomic units for Boron, e.g. atoms/ion, and then convert it to volumetric units for BN. The CRDS sputter yields for BN, found in this way, are shown in the right of Figure 4. Note that our conversion from the measured boron atomic yield to volumetric $\mathrm{BN}$ yield assumes that all sputter products are $\mathrm{B}$ and $\mathbf{N}$ atoms. This assumption is important because if some of the sputter products are clusters (e.g. $\mathrm{B}_{\mathrm{x}} \mathrm{N}_{\mathrm{y}}$ or $\mathrm{B}_{\mathrm{z}}$ ), then these species would not be detected by the CRDS; therefore, the CRDS sputter yields for BN should be considered as lower bounds.

The right of Figure 4 also shows BN sputter yields by QCM and weight loss from our past work $^{4,7,37,38}$ as well as measurements from other researchers ${ }^{39-43}$. The sputter yield from QCM measurements at CSU are plotted such that the lower reach of each error bar represents the lower possible bound of BN yield (i.e. the actual condensable measurement, which would be the true yield if all sputtering was as clusters), while the upper reach of each error bar represents the upper possible bound of BN yield (i.e. a value found by scaling the measured yield by 2.30 corresponding to a case where all sputtering is as boron and nitrogen atoms but the QCM detects only the boron; see also References 4 and 38). The CSU sputter yield measurements by CRDS (this work) and QCM in $2007^{37}$ and in $2008^{4}$ are self-consistent if one assumes that most of the sputtering is as atoms (in which case the lower-bound CRDS yields are slight-underestimates, and one should adopt QCM yields close to the top of the error-bar ranges). In this case the CSU 2008 weight loss measurements ${ }^{4}$ are also close to self-consistent (they are slight overestimations), while past CSU weight loss measurements are significant underestimations (perhaps due to ion implantation).
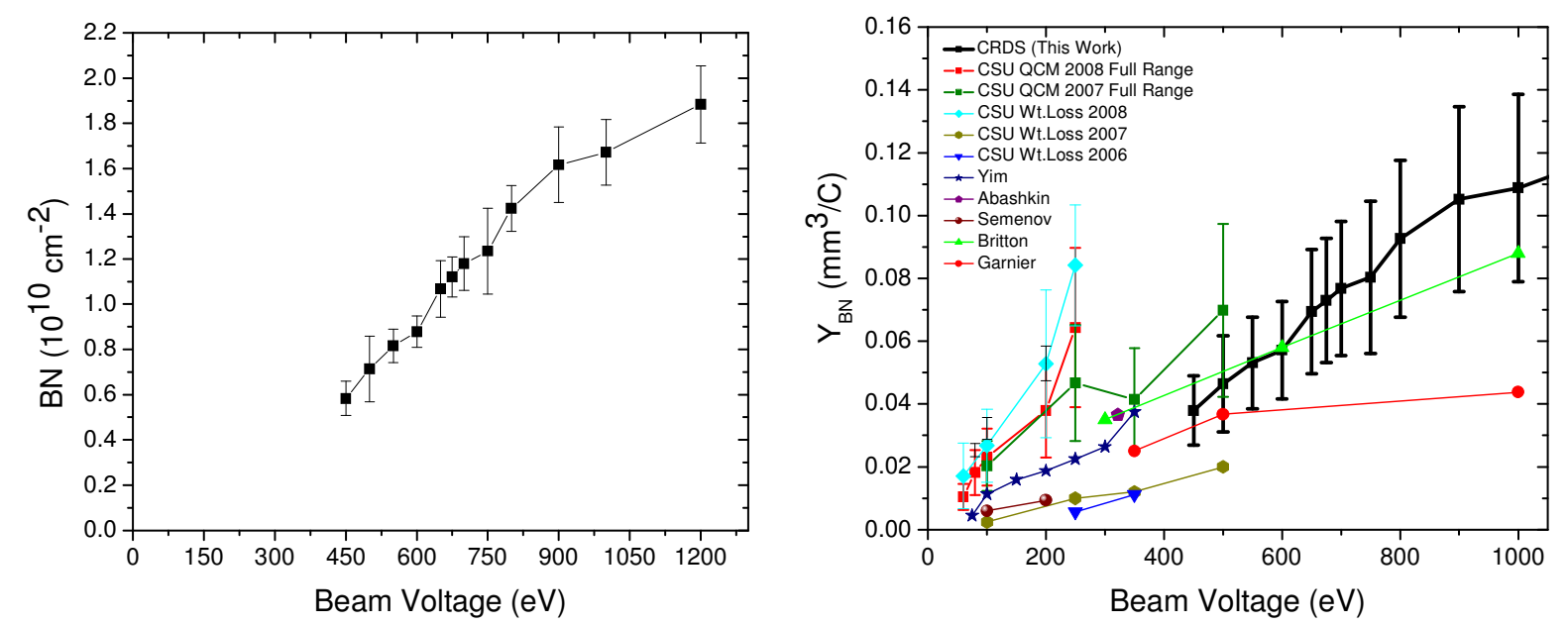

Figure 4. Left: Path-integrated number density of BN versus ion beam energy. Right: BN sputter yield versus ion energy (see text).

\section{Development of CW-CRDS System}

\section{A. Experimental Layout}

The pulsed-CRDS system discussed above guides the development of a cw-CRDS system. Implementation of cw-CRDS system provides improved sensitivity relative to pulsed-CRDS as will be needed for future in situ thruster measurements. In order to obtain exponential ring-down signals (as described by equation (1)) using the cw-CRDS system, one needs to appropriately control the laser and cavity frequencies. The basic differences between pulsedand cw-CRDS relate to the relative linewidths of the laser and cavity. In pulsed-CRDS the laser bandwidth is $\sim 2$ $\mathrm{GHz}$ which overlaps many cavity transmission peaks; cavity peaks are spaced by the free-spectral range, i.e. the mode-spacing, of the ring-down cavity $(\mathrm{FSR}=c / 2 l=200 \mathrm{MHz}$ for $l=75 \mathrm{~cm}$ ). Therefore, in pulsed experiments the laser will have some coupling to the cavity regardless of the precise laser tuning. On the other hand, in cw-CRDS the laser linewidth is $\sim 3 \mathrm{MHz}$ and thus much narrower than the cavity peaks; in the cw-case the laser will generally not be transmitted into, or through, the cavity. In the present experiments we simply scan the laser until we achieve 
a resonance with the cavity, after which we use an acousto-optic modulator (AOM) to extinguish the incoming laser beam. Following extinction of the beam, the light within the cavity decays and a ring-down signal can be measured. In our future work we may scan the position of the rear cavity-mirror via a piezoelectric (PZT) stack (to bring the cavity into resonance with the laser at any laser wavelength) and again use the acousto-optic modulator (AOM) to extinguish the laser beam.

A schematic diagram of the BN CRDS set-up that we are developing is shown in Figure 5. In this discussion, the emphasis is on the optical detection system itself, issues related to implementation for Hall thruster studies and large vacuum chambers are briefly addressed in Section IVC. The BN detection scheme is based on probing absorption lines in the vicinity of $249 \mathrm{~nm}$ as discussed above. The cw- light sources is a commercially available frequency-quadrupled external cavity diode laser. The external cavity diode laser has output of $\sim 10 \mathrm{~mW}$, linewidth of $\sim 3 \mathrm{MHz}$, and can be continuously mode-hop-free tuned over $\sim 30 \mathrm{GHz}$ in our wavelength region of interest. The laser output is mode-matched to the cavity using a pair of lenses. A threshold circuit is used to monitor the cavity transmission, and when a preset level is reached the AOM is triggered and used to extinguish the input light. The AOM is designed for 244-260 nm by NEOS Technologies, Inc, with a diffraction efficiency of $70 \%$ for linear polarized light perpendicular to acoustic propagation. The ring-down cavity is based on a pair of high reflective mirrors having $\mathrm{ROC}=1-\mathrm{m}$ and reflectivity of $\mathrm{R} \sim 99.5 \%$ for $249 \mathrm{~nm}$. The cavity length in the current setup is $66 \mathrm{~cm}$. Ring-down signals are detected with a photomultiplier tube (Hamamatsu R9110 with a DA-type socket C7247). Signals are collected to computer using an A/D board (Adlink PCI-9812 with gain error less than $\pm 1.5 \%$.)

Figure 6 plots the cavity transmission as the laser wavelength is swept and illustrates the mode-matching. The regularly-spaced peaks (labeled in the plot) correspond to the fundamental $\mathrm{TEM}_{q 00}$ cavity transmission modes. In this experiment the peaks exceeded the input maximum of the board, 5V. Other cavity peaks originating from higher-order cavity modes can also be seen. These peaks are from the higher-order non-fundamental modes, i.e. $\mathrm{TEM}_{q m n}$ modes that are not $\mathrm{TEM}_{q 00}$. Current work seeks to reduce coupling to these modes, however, even in the current setup they are sufficiently small to allow unambiguous thresholding on the fundamental modes (e.g. for a threshold level in the $2-5 \mathrm{~V}$ range).

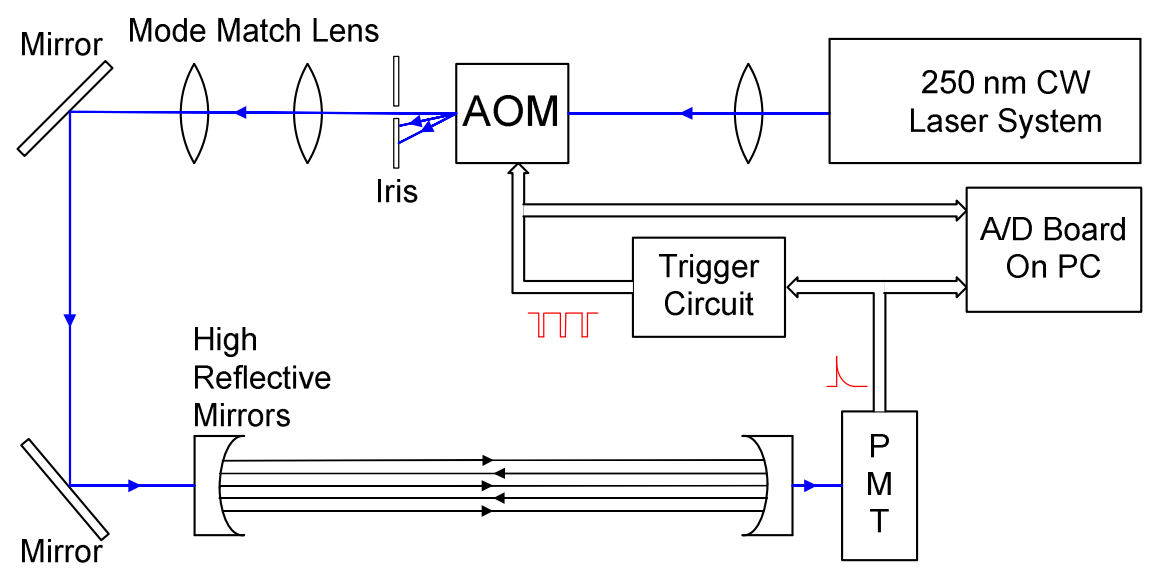

Figure 5. Schematic of cw-CRDS setup. 


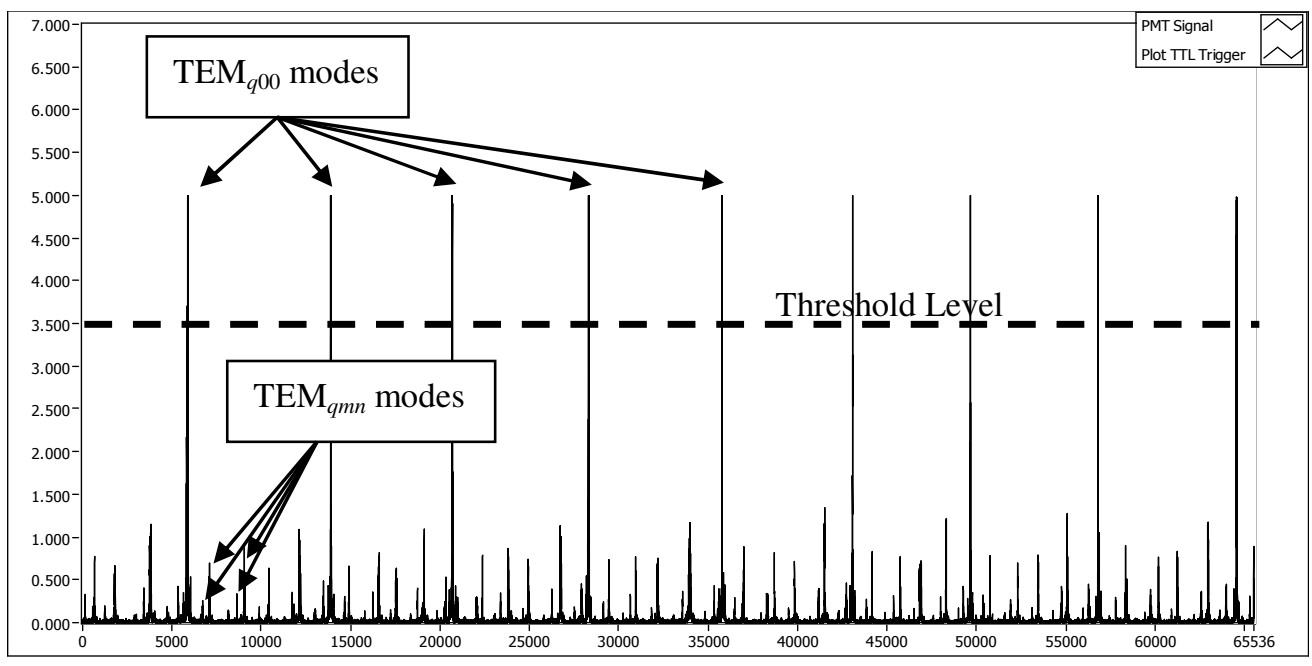

Figure 6. Transmission of laser light through optical cavity as laser frequency is swept. Examples of fundamental and higher-order modes are shown along with a possible trigger level.

We have not yet coupled the cw-CRDS system with the sputter diagnostic chamber, nonetheless we desire to characterize system performance and therefore we characterize the current sensitivity of the system by making a measurement of the mirror loss $(1-R)$ based on the ring-down times using eqn. (1). Figure 7 shows the mirror reflectivity determined in this manner, assuming that each mirror has the same loss. Each data point corresponds to 10 -s of measurement corresponding to the average of roughly 300 ring-down signals. The reflectivity is approximately consistent with manufacturer's specification of $>99.5 \%$. More interesting is to examine the noise in the reflectivity as a means to look at the sensitivity. For this data, the noise in the reflectivity is approximately 10 ppm for measurement times of 10 s of seconds. The cavity loss, equal to 1-R, has the same noise level, so the current configuration allows loss measurements of $\sim 10 \mathrm{ppm}$ for measurement times of $10 \mathrm{~s}$ of seconds. The sensitivity is further quantified below.

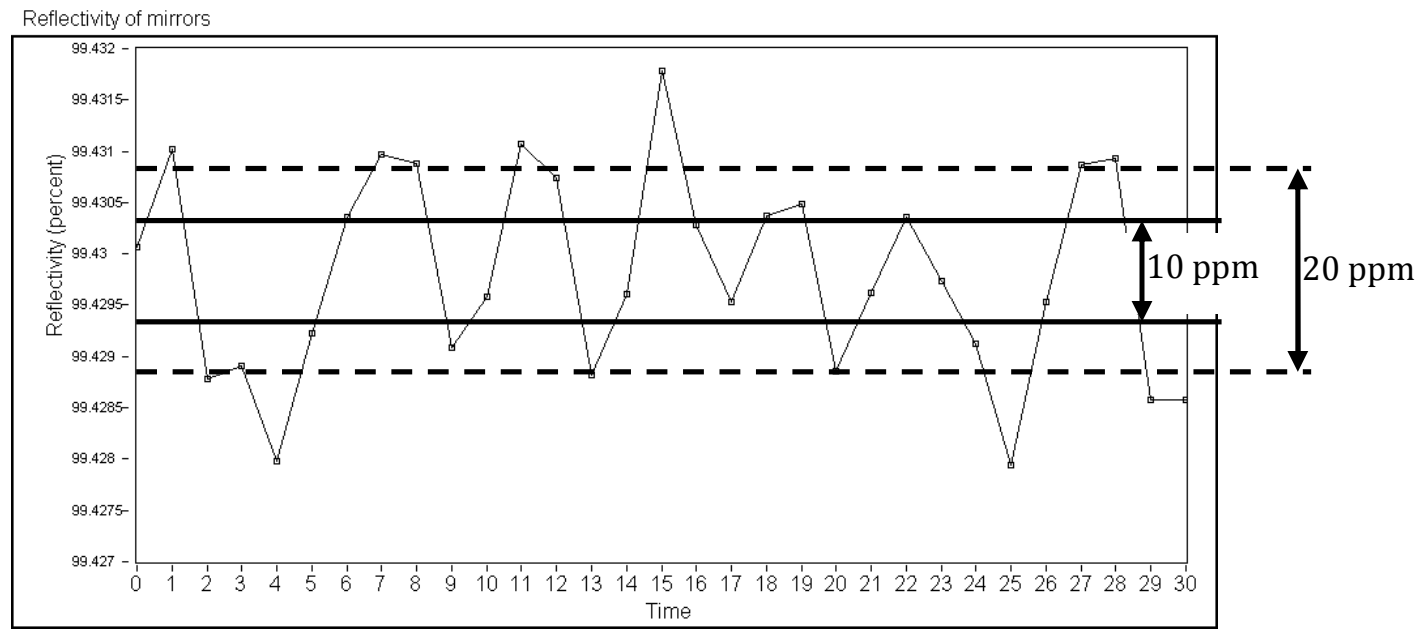

Figure 7. Mirror reflectivity as measured by CRDS versus time, where each time increment corresponds to $10 \mathrm{~s}$ (or approximately 300 ring-down signals).

\section{B. Sensitivity and Signal-to-Noise Estimation}

We also gauge the system sensitivity by examining the noise and its dependence on acquisition time. Figure 8 shows a direct experimental measurement of the minimum detectable absorbance where we compute the minimum detectable absorbance, $A b s_{\text {Min }}$, as ${ }^{35}$ : 


$$
A b s_{M i n} \equiv \frac{\Delta \tau}{\tau}(1-R)
$$

where $\Delta \tau$ is the uncertainty (noise) in measurement of ring-down time $\tau$, for which we use the standard error in measurement of $\tau$. The standard error represents the expected standard deviation between the measured estimates and true-value, and is computed as the standard deviation divided by the square-root of the number of measurements. As can be seen in Figure 8, for short measurement times, e.g. 10-s, the minimum detectable absorbance in roughly $20 \mathrm{ppm}, 3 \mathrm{ppm}$ can be achieved in approximately 3 minutes, and sub-ppm sensitivity requires approximately 20-30 minutes of collection time. In a thruster experiment one could switch back-and-forth between resonant and non-resonant wavelengths, for example every 1-minute, in order to normalize away any drift effects.

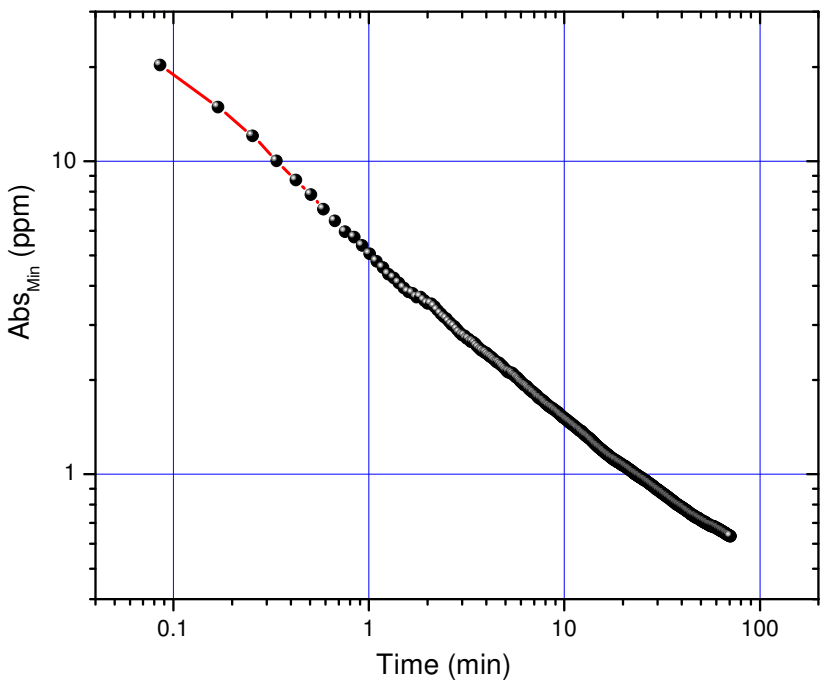

Figure 8. Minimum detectable absorbance as function of acquisition time.

Given the relatively poor reflectivity of mirrors in the $249 \mathrm{~nm}$ region, our pulsed-CRDS provides measurement sensitivity of $\sim 50 \mathrm{ppm}$ for measurement times of minutes. The current cw-CRDS system allows a sensitivity of approximately $3 \mathrm{ppm}$ for measurement times of 3 minutes and better sensitivity for longer times. Comparing the two configurations, the cw-CRDS system currently has approximately 15 times better sensitivity than pulsed-; however, an improvement of greater than a factor of 100 should be expected (see Reference 35 and therein). We can readily achieve improved sensitivity by increasing our acquisition rate, which is about $30 \mathrm{~s}^{-1}$ in the current system but can readily be increased to about $300 \mathrm{~s}^{-1}$ by increasing laser scan-speed, and to kHz rates by using a PZT on a cavity mirror (and changing A/D board). In related work for end-point detection of sputtered manganese, we have demonstrated higher time response and acquisition rates ${ }^{44}$. Finally, the noise in fitting individual ring-down signals is currently $\sim 3 \%$ and needs to be improved via mode-matching and electronics.

To gauge our expected signal-to-noise ratio (SNR) in thruster measurements, we compare the anticipated detection limit with the expected value in the thruster plume. Based on scaling of wall erosion rates modeled by Yim et al. ${ }^{45}$, we predict a path-integrated number density at the thruster exit plane of boron of $10^{9} \mathrm{~cm}^{-2}$ to $10^{10} \mathrm{~cm}^{-2}$, corresponding to peak optical absorbance signals of approximately 30-300 ppm. Therefore, the current cw-CRDS system could measure boron in the plume of an operating thruster with high signal-to-noise for integration times of 10s of seconds to several minutes (depending on exact parameters). Table 2 shows expected signal-to-noise ratios for several detection systems and boron concentrations in the thruster plume. We are assuming a similar linewidth to that measured in our pulsed-CRDS experiments $(\sim 0.007 \mathrm{~nm})$. In summary, the current $\mathrm{cw}$-CRDS system with longer integration times already should yield the needed sensitivity. Relatively straightforward improvements, as summarized above, can increase signal-to-noise or reduce measurement times. A projected system based on prismreflectors (described below) should yield even higher sensitivity. It is also interesting that (especially for long measurement times) the detection limit of our future CRDS systems should be sufficiently low, that if the thruster tests do not yield a measurable signal, then we will have confirmed that the boron concentrations $\left(\sim 10^{6} \mathrm{~cm}^{-3}\right)$ and associated erosion rates $(\sim 0.1 \mu \mathrm{m} / \mathrm{hr})$ are low enough to correspond to very long thruster lifetimes $(>9,000$ hours). 
Table 2. Predicted signal-to-noise ratios for CRDS detection systems and thruster concentrations.

\begin{tabular}{|c|c|c|c|c|c|}
\hline Detection System & $\begin{array}{l}\text { Integration time } \\
\text { (s) }\end{array}$ & $\begin{array}{l}\text { Abs } \operatorname{Min}(p p m) \\
\text { Fixed } \\
\text { Frequency }\end{array}$ & $\begin{array}{l}\text { Abs }{ }_{\text {Min }}(p p m) \\
\text { Scanned } \\
\text { Frequency }\end{array}$ & $\begin{array}{l}\text { S:N (for [B] } \\
\left.10^{9} \mathrm{~cm}^{-2}\right)\end{array}$ & $\begin{array}{l}\mathrm{S}: \mathrm{N}(\text { for }[\mathrm{B}]= \\
\left.10^{10} \mathrm{~cm}^{-2}\right)\end{array}$ \\
\hline Pulsed CRDS (current) & 180 & 50 & 50 & 0.6 & 6 \\
\hline \multirow[t]{3}{*}{ cw-CRDS (current) } & 10 & 12 & 36 & 1 & 10 \\
\hline & 180 & 3 & 10 & 3 & 30 \\
\hline & 1800 & 1 & 3 & 10 & 100 \\
\hline \multirow[t]{2}{*}{ cw-CRDS (improved) } & 10 & 1.2 & 4 & 7.5 & 75 \\
\hline & 180 & 0.3 & 1 & 30 & 300 \\
\hline Prism-Based cw-CRDS & 1 & 0.1 & 0.3 & 100 & 1000 \\
\hline
\end{tabular}

Notes:

1. We assume detection limit scales as integration time to the exponent -0.5 .

2. The $\mathrm{cW}$-CRDS (improved) assumes acquisition rate of $300 \mathrm{~s}^{-1}$ rate and $3 \mathrm{x}$ improved noise in individual ringdowns.

3. For cw-CRDS we assume that frequency scanning reduces sensitivity by a factor of 3 owing to need to switch between on- and off- resonant wavelengths and bin data and construct lineshapes.

Another avenue to improve the sensitivity relates to the mirror reflective loss. As discussed above, mirrors in the $249 \mathrm{~nm}$ region have maximum reflectivity of about 0.997 , corresponding to $3000 \mathrm{ppm}$ loss. A new approach to CRDS involves the use of optical cavities, based upon a pair of Brewster Angle retroreflectors. As shown in Figure 9, the optical ring cavity is folded by light entering a prism at or very near Brewster's angle (where there is very low loss for p-polarized light), undergoes two total internal reflections, and then leaves parallel to the input beam ${ }^{38}$. For our experiments the motivation for the prism cavity is that when made from $\mathrm{CaF}_{2}$ the optical loss of the prisms can be extremely low. $\mathrm{CaF}_{2}$ is particularly promising for the UV since it is known that the scattering loss is only a few percent that of fused silica ${ }^{46}$ and is transparent deep in the UV. Based upon recent scattering measurements, we can predict that prism retro-reflector loss as low as $50 \mathrm{ppm}$ per reflection should be obtainable at $249 \mathrm{~nm}$, nearly two orders of magnitude lower than the lowest loss dielectric mirrors available in this UV region. The use of the prism cavity should provide significant sensitivity improvement and will be presented in our future submissions.

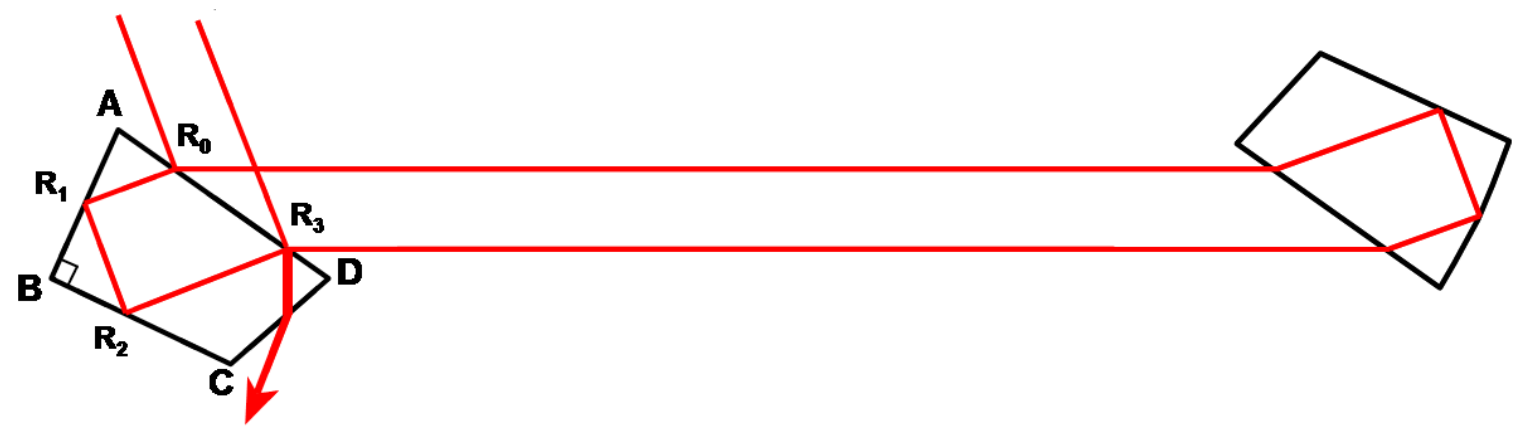

Figure 9. Schematic of Brewster Prism Cavity.

\section{Conclusion}

We have described the development of CRDS detection for quantitative measurements of sputtered boron nitride based on boron atom absorption at $249 \mathrm{~nm}$. We have shown results of pulsed-CRDS detection of BN targets including variation of boron concentration versus ion energy. Knowing the current distribution of the ion beam, and making certain assumptions on the velocity profile and shape of the differential sputter yield allows one to infer total sputter yields. The total sputter yields found in this manner have been compared with results from other researchers. We note that the BN yields inferred from the CRDS should be treated as lower bounds (since they may miss cluster species, though we can account for atomic nitrogen paired with atomic boron) and we find good self-consistency with CSU QCM measurements from 2007 and 2008 and with our most recent 2008 weight loss measurements.

In addition to basic material characterization, we are focused on development of a sputter erosion sensor based on cw-CRDS. In comparison to existing diagnostic methods, the cw-CRDS sensor should afford several key advantages including the possibility of accelerated (near real-time) measurements of thruster erosion performed in 
situ. Clearly, such a diagnostic has high potential for accelerated thruster lifetime testing and as a tool for thruster design. Development of a cw-CRDS system at $249 \mathrm{~nm}$ has several challenges, the foremost of which is the relatively high reflective loss of mirrors at this wavelength. Nonetheless, we are showing success in the development of a cw-CRDS system and the system in its current state should have the needed sensitivity for measurements at expected thruster conditions if relatively long integration times (minutes) are used. Approaches for improved sensitivity, including increased sampling rate, and the use of novel prism retro-reflectors have also been presented.

Implementation of the cw-CRDS sensor for thruster measurements will have several challenges beyond the optical sensitivity of the detection system. On the hardware side, challenges include delivery of the laser beam into the chamber and ability to maintain the cavity alignment and mirror cleanliness. We have recently demonstrated some of these aspects using single-mode fiber optic delivery ${ }^{44}$ and a series of baffles to shield the mirrors and maintain their reflectivity. For $249 \mathrm{~nm}$, conventional single-mode fibers are unavailable and we are considering the use of photonic crystal fibers. We may also implement motorized mirror mounts for the beam delivery and cavity alignment. Other challenges relate to interpretation of the signal and its relation to erosion rate. Two key issues are relation of the boron sputter signal to the overall BN sputter signal and our ability to relate the measured number densities to fluxes (note that erosion rate is proportional to the flux of sputtered particles). For the former, we will leverage QCM and weight-loss measurements of BN currently being performed at CSU. Several approaches are being considered for the latter, including simple numerical estimates based on mean velocities and laser measurements of velocities. A final issue is considering the possibility that a fraction of the sputtered boron will be ionized (making it undetectable) before it reaches the measurement region; initial numeric estimates show that at most $\sim 10 \%$ of the particles will be ionized. While challenges remain, we view that the cw-CRDS approach has high potential to address the critical need of accelerated in situ thruster lifetime testing.

\section{Acknowledgments}

The researchers acknowledge technical help from Mr. J. Topper (M.Sc. student at CSU), Dr. B. Rubin (Postdoctoral researcher at CSU), and Dr. J. Williams (Assistant Professor at CSU).

\section{References}

${ }^{1}$ Ahedo E. et al. 2007 Simulation of wall erosion in Hall thrusters, $30^{\text {th }}$ International Electric Propulsion Conference, Florence, Italy, IEPC-2007-067

${ }^{2}$ Cheng S.Y. et al. 2007 Modeling of Hall thruster lifetime and erosion mechanisms, $30^{\text {th }}$ International Electric Propulsion Conference, Florence, Italy, IEPC-2007-250

${ }^{3}$ Yim J.T., Boyd I.D., Keidar M. 2007, "Hall Thruster Erosion Prediction Using A Hydrodynamic Plasma Model And Sputtering Simulation", $30^{\text {th }}$ International Electric Propulsion Conference, Florence, Italy, IEPC-2007-034

${ }^{4}$ Topper J., Rubin B., Farnell C., Yalin A.P. 2008 Preliminary Results of Low Energy Sputter Yields of Boron Nitride due to Xenon Ion Bombardment, 44th AIAA/ASME/SAE/ASEE Joint Propulsion Conference, Hartford, CT, AIAA-08-5092

${ }^{5}$ Garner C. E., Brophy J. R., Polk J. E., and Pless L. C. 1995 A 5730-Hr Cyclic Endurance Test of the SPT-100, AIAA-95-2667

${ }^{6}$ Anderson, J, Goodfellow, K., Polk, J., and Shotwell, H. 1999 Results of an On-going Long Duration Ground Test of the DS-1 Flight Space Engine, AIAA-99-2858, $35^{\text {th }}$ Joint Propulsion Conference, Los Angeles, CA

${ }^{7}$ Yalin A. P., Surla V., Farnell C., Butweiller M., and Williams J. D. 2006 Sputtering studies of multi-component materials by weight loss and cavity ring-down spectroscopy, 42nd AIAA Joint Propulsion Conference, Sacramento, CA

${ }^{8}$ Chiplonkar V. T. and Rane S. R. 1965 Dependence of angular distribution of sputtering by positive ions from metal targets on the impact angle, Indian J. Pure Appl Phys 3161

${ }^{9}$ Tsuge H. and Esho S. 1981 Angular distribution of sputtered atoms from polycrystalline metal targets, J. Appl. Phys. 52 4391-95

${ }^{10}$ Kolasinksi R. D., Polk J. E., Goebel D. and Johnson L. J. 2006 Carbon sputtering yield measurements at grazing incidence, 42nd AIAA/ASME/SAE/ASEE Joint Propulsion Conference (Sacramento, CA) AIAA 2006-4337

${ }^{11}$ Zoerb K. A., Williams J. D., Williams D. D., and Yalin A. P. 2005 Differential sputtering yields of refractory metals by xenon, krypton, and argon ion bombardment at normal and oblique incidences, 29th International Electric Propulsion Conference (Princeton, NJ) IEPC-2005-293 
${ }^{12}$ Wickersham C. E. and Zhang Z 2005 Measurement of angular emission trajectories for magnetron-sputtered tantalum, J. Electronic Materials 34

${ }^{13}$ Kundu S., Ghose D., Basu D., and Karmohapatro S. B. 1985 The angular distribution of sputtered silver atoms, Nuclear Instruments and Methods in Physics Research B 12 352-57

${ }^{14}$ Wucher A. and Reuter W. 1988 Angular distribution of sputtered particles from metals and alloys, J. Vac. Sci. Tech. A 6(4) 2316-18

${ }^{15}$ Shutthanandan V., Ray P., Shivaparan N., Smith R., Thevuthasan T., and Mantenieks M. 1997 On the measurement of low-energy sputtering yield using Rutherford backscattering spectrometry, 25th International Electric Propulsion Conference (Cleveland, OH) IEPC-97-069

${ }^{16}$ Mantenieks M., Foster J., Ray P., Shutthanandan S., and Thevuthasan T. 2001 Low energy xenon ion sputtering yield measurements, 27th International Electric Propulsion Conference (Pasadena, CA) IEPC-01-309

${ }^{17}$ Andersen N., B. Andresen, and E. Veje 1982 Atomic excitations in sputtering processes, Radiation Effects 60119 127

${ }^{18}$ Doerner R.P., Whyte D.G., and Goebel D.M. 2003 Sputtering yield measurements during low energy xenon plasma bombardment, J. App. Phys. 93(9) 5816-5823

${ }^{19}$ Pellin M.J., Wright R.B., and Gruen D.M. 1981 Laser fluorescence spectroscopy of sputtered zirconium atoms, $J$. Chem. Phys. 74 6448-6457

${ }^{20}$ Bay H.L. 1987 Laser induced fluorescence as a technique for investigations of sputtering phenomena, Nuclear Instruments and Methods B18 430-445

${ }^{21}$ Young C.E., et al.1984 Velocity and electronic state distributions of sputtered $\mathrm{Fe}$ atoms by laser-induced fluorescence spectroscopy, J. Vac. Sci. Technol. A 2(2) 693-697

${ }^{22}$ Nicolussi G., et al. 1995 Formation of metastable excited Ti and Ni atoms during sputtering, Phys. Rev. B 51(14) 8779-8788.

${ }^{23}$ Goehlich A. 2001 Investigation of time-of-flight and energy distributions of atoms and molecules sputtered from oxygen-covered metal surfaces by laser techniques, Appl. Phys. A. 72 523-529

${ }^{24}$ Staudt C., et al. 2002 Sputtering of Ag atoms into metastable excited states, Phys. Rev. B 66085415 1-12

${ }^{25}$ Dyshlyuk E.N. and Gorshkov A. 2006 Spectroscopic Investigation of a Hall Thruster Ceramic Acceleration hannel Erosion Rate 42nd AIAA/ASME/SAE/ASEE Joint Propulsion Conference \& Exhibit, Sacramento, California AIAA 2006-4660

${ }^{26}$ Karabadzhak G.F. et al. 2005 Evaluation of Impurity Composition and Content in the TAL at Various Operating Regimes International Electric Propulsion Conference, Princeton University, IEPC-2005-147

${ }^{27}$ Pagnon D., Lasgorceix P., Touzeau M. 2004 Control of the ceramic erosion by Optical Emission Spectroscopy: Results of PPS1350-G measurements Fourth ISPC, Cagliari, Sardinia

${ }^{28}$ Surla V., Wilbur P.J., Johnson M., Williams J.D., and Yalin A.P. 2004 Sputter erosion measurements of Titanium and Molybdenum by cavity ring-down spectroscopy, Review of Scientific Instruments 75(9) 3025-3030

${ }^{29}$ Yalin A.P., Surla V., Butweiller M., and Williams J.D. 2005 Detection of Sputtered Metals using Cavity RingDown Spectroscopy, Applied Optics 44, 30 6496-6505

${ }^{30}$ Yalin A.P. and Surla V. 2005 Velocity Measurements by Cavity Ring-Down Spectroscopy, Optics Letters, 30, 3219

${ }^{31}$ Surla V. and Yalin A.P. 2007 Differential Sputter Yield Measurements using Cavity Ring-Down Spectroscopy Applied Optics 46, 19 p.3978-

${ }^{32}$ Yalin A.P. et al. 2007 Boron Nitride Sputter Erosion Measurements by Cavity Ring-Down Spectroscopy Electric Propulsion Conference Florence, Italy IEPC-2007-075

${ }^{33}$ Busch, K.W., and Busch, M.A., "Cavity-Ringdown Spectroscopy," ACS Symposium Series, Vol. 720, 1999.

${ }^{34}$ Berden, G., Peeters, R., and G. Meijer 2000 Cavity Ring-Down Spectroscopy: Experimental Schemes and Applications, International Reviews in Physical Chemistry, Vol.19, No.4, p. 565-607, 2000.

${ }^{35}$ Zalicki, P. and Zare, R.N. 1995 Cavity ring-down spectroscopy for quantitative absorption measurements, Journal of Chemical Physics, Vol. 102, No.7, p. 2708-17

${ }^{36}$ Yalin, A.P., and Zare, R.N., 2002 Effect of Laser Lineshape on the Quantitative Analysis of Cavity Ring-Down Signals Laser Physics, Vol.12, No.8, p. 1065-1072

${ }^{37}$ Yalin, A.P. at al. 2007 Differential Sputter Yields Of Boron Nitride, Quartz, and Kapton Due to Low Energy Xe ${ }^{+}$ Bombardment, 43rd AIAA/ASME/SAE/ASEE Joint Propulsion Conference

${ }^{38}$ Rubin B., Topper J.L. Yalin A.P. 2007 Total and Differential Sputter Yields Of Boron Nitride Measured by Quartz Crystal Microbalance and Weight Loss, $30^{\text {th }}$ International Electric Propulsion Conference, Florence, Italy, IEPC-2007-074 
${ }^{39}$ Yim, J. 2008 Computational Modeling of Hall Thruster Channel Wall Erosion, Ph.D. Dissertation, Dept. of Aerospace Engineering, University of Michigan, MI

${ }^{40}$ Garnier Y et al. 1999 Low-energy xenon ion sputtering of ceramics investigated for stationary plasma thrusters J. Vac. Sci. Tech. A 17 (6) pp. 3246-3254

${ }^{41}$ Semenov A., Shkarban, I., 1991 Ion Beam Sputtering of the Surfaces of Ion and Plasma Sources, Rocket and Space Engineering: Rocket Engines and Power Plants, No.3, pp.42-53 (in Russian).

${ }^{42}$ Abashkin, V., Gorshkov, O., Lovtsov, A., and Shagaida, A. 2007 Analysis of Ceramic Erosion Characteristic in Hall-Effect Thruster with Higher Specific Impulse $30^{\text {th }}$ International Electric Propulsion Conference, Florence, Italy, IEPC-2007-133

${ }^{43}$ Britton, M. Waters, D., Messer, R., Sechkar, E., and Banks, B. 2002 Sputtering Erosion Measurements on Boron Nitride as a Hall Thruster Material, NASA/TM 2002-211837

44 Tao L., Yalin A.P., Yamamoto N. 2008 Cavity Ring-Down Spectroscopy Sensor for Ion Beam Etch Monitoring and End-Point Detection of Multilayer Structures, Accepted for Publication in Review of Scientific Instruments

${ }^{45}$ Yim, J.T., Keidar, M., and Boyd. I.D., "An investigation of factors involved in Hall thruster wall erosion modeling, AIAA-2006-4657, 42nd Joint Propulsion Conference, Sacramento, CA, Jul 2006.

${ }^{46}$ G. Engel, W. B. Yan, J. B. Dudek, K. K. Lehmann, and P. Rabinowitz, "Ring-down spectroscopy with a Brewster's angle prism resonator," in Laser Spectroscopy XIV International Conference, R. Blatt, J. Eschner, D. Leibfried, and F. Schmidt-Kaler, eds. (World Scientific, Singapore, 1999), pp. 314-315. 